\title{
Electron-Phonon Interaction and Antiferromagnetic Correlations
}

\author{
G. Sangiovanni, ${ }^{1}$ O. Gunnarsson, ${ }^{1}$ E. Koch, ${ }^{2}$ C. Castellani, ${ }^{3}$ and M. Capone $e^{4,3}$ \\ ${ }^{1}$ Max-Planck Institut für Festkörperforschung, Heisenbergstrasse 1, D-70569 Stuttgart, Germany \\ ${ }^{2}$ Institut für Festkörperforschung, Forschungszentrum Jülich, 52425 Jülich, Germany \\ ${ }^{3}$ Dipartimento di Fisica Università di Roma "La Sapienza" piazzale Aldo Moro 5, I-00185 Roma, Italy \\ ${ }^{4}$ INFM-SMC and Istituto dei Sistemi Complessi, Consiglio Nazionale delle Ricerche, Via dei Taurini 19, I-00185 Roma, Italy \\ (Received 24 February 2006; published 26 July 2006; publisher error corrected 28 July 2006)
}

\begin{abstract}
We study effects of the Coulomb repulsion on the electron-phonon interaction (EPI) in the HolsteinHubbard model, using the antiferromagnetic (AF) dynamical mean-field approximation. AF correlations strongly enhance EPI effects on the electron Green's function with respect to the paramagnetic correlated system, but the net effect of the Coulomb interaction is a moderate suppression of the EPI. Doping leads to additional suppression. In contrast, the Coulomb interaction strongly suppresses EPI effects on phonons, but the suppression weakens with doping.
\end{abstract}

DOI: 10.1103/PhysRevLett.97.046404

PACS numbers: 71.38. $-\mathrm{k}, 71.27 .+\mathrm{a}, 74.72 .-\mathrm{h}$

There are strong experimental indications that the electron-phonon interaction (EPI) plays a substantial role for properties of high- $T_{c}$ cuprates [1,2], and that it even can lead to formation of small polarons for undoped cuprates [3]. The Coulomb interaction in the copper-oxide plane is expected to strongly suppress charge fluctuations. This is often described in the Hubbard or $t-J$ [4] models, for which important phonons couple to such charge fluctuations [5]. One might therefore actually expect the Coulomb interaction to strongly suppress the EPI. This was indeed found with dynamical mean-field theory [6,7] calculations in the paramagnetic phase ( $P$-DMFT) [8-10]. On the other hand, calculations for the undoped antiferromagnetic $t-J$ model using the self-consistent Born approximation (SCBA) [11], or approximations going beyond the SCBA [12], found that the interaction with antiferromagnetic fluctuations enhances EPI effects on the electron Green's function.

The half-filled Hubbard model becomes an insulator for large $U$. In the $P$-DMFT the only mechanism for this is the quasiparticle weight $Z$ going to zero [7]. Such a reduction of $Z$ tends to strongly suppress the EPI [10,11], which is an important reason for the small effects of the EPI in the $P$-DMFT. By allowing for antiferromagnetism (AF) in the DMFT [13-15], it is possible to have an insulating state although $Z$ remains finite. This suggests that it is important to allow for $\mathrm{AF}$ when describing the EPI, i.e., using an AF-DMFT. This is also suggested by the fact that in the SCBA, the enhancement of the EPI grows with the value of the exchange constant $J$ [11], i.e., with the importance of the AF.

Here we use the AF-DMFT formalism to study the effects of AF correlations in the Holstein-Hubbard model at both half filling and, for the first time, other fillings (finite doping). As a comparison, we also perform $P$-DMFT calculations. We find that the effects of the EPI on the electron Green's function are neither strongly suppressed nor enhanced by the Coulomb interaction. While previous work addressed [11,12] the half-filled case, the present formalism can treat doped systems, which are of particular interest. We show that the EPI remains important for the electron Green's function as long as AF is important. Because of the weakening of AF correlations as the system is doped, we find that doping reduces the effects of the EPI on the electron Green's function and it weakens the tendency to polaron formation, in agreement with experimental results [3].

We also calculate the renormalization of the phonon spectrum. For the undoped system, we find that the Coulomb repulsion very strongly suppresses the renormalization. As the system is doped, however, the renormalization increases. This is the opposite behavior to what we find for the electron Green's function. The width and softening of the phonon spectral function is often used to estimate the strength of the EPI [16]. These results show that for strongly correlated systems this approach may strongly underestimate the EPI $[17,18]$.

We study the Holstein-Hubbard model

$$
\begin{aligned}
H= & -t \sum_{\langle i j\rangle \sigma}\left(c_{i \sigma}^{\dagger} c_{j \sigma}+\text { H.c. }\right)+U \sum_{i} n_{i \uparrow} n_{i \downarrow} \\
& +\omega_{0} \sum_{i} b_{i}^{\dagger} b_{i}+g \sum_{i}\left(n_{i}-1\right)\left(b_{i}+b_{i}^{\dagger}\right),
\end{aligned}
$$

where $c_{i \sigma}^{\dagger}$ creates an electron with spin $\sigma$ on site $i$ and $b_{i}$ a phonon on site $i, t>0$ is a hopping integral, $U$ the Coulomb interaction between two electrons on the same site, $\omega_{0}$ is the phonon energy, $g$ a coupling constant, and $n_{i}$ measures the number of electrons on site $i$. We assume an infinite-coordination Bethe lattice with the half bandwidth $D$ and the density of states (DOS) $N(\varepsilon)=\left(2 / \pi D^{2}\right) \times$ $\sqrt{D^{2}-\varepsilon^{2}}$. We define a dimensionless coupling constant $\lambda=g^{2} /\left(\omega_{0} D\right)$. For large $U$ the Hubbard model is approximately equivalent to the $t$ - $J$ model. For a two-dimensional (2D) lattice, these models are related via $J / t=D / U$.

We solve the DMFT equations for the temperature $T=$ 0 . The associated impurity problem is solved using the 
Lanczos method. The Hilbert space is limited by only allowing up to $N_{\text {ph }}$ phonons, where $N_{\text {ph }} \sim 30$ depends on the parameters. The energies of and couplings to the bath levels are determined from a continued fraction expansion [19] for the large $U$ half-filled case and otherwise by a fit of the cavity Green's function on the imaginary axis [20]. We use up to 25 bath levels.

In the following we focus on the quasiparticle weight $Z$. As discussed above, $Z$ is expected to be crucial for the EPI, and the description of $Z$ in the AF-DMFT is therefore important. Furthermore, our criterion for polaron formation is that $Z$ is exponentially small. Figure 1 shows $Z_{0}$, calculated for $\lambda=0$, as a function of $U$ for the half-filled Hubbard model. The model is an insulator in the AFDMFT and for $U \gtrsim 3 D$ in the $P$-DMFT [15]. While $Z_{0}$ drops to zero very quickly with $U$ in the $P$-DMFT, it remains finite in the AF-DMFT. Figure 1 also shows $Z_{0}$ as a function of $J$ for the (2D) $t-J$ model according to the SCBA, which agrees well with exact diagonalization for small clusters [21]. For $U / D$ values where the Hubbard and $t$ - $J$ models are approximately equivalent, AF-DMFT and SCBA agree rather well. This good description of $Z_{0}$ suggests that AF-DMFT may describe the EPI well.

We first discuss the results for a weak EPI $(\lambda \lesssim 0.1)$. For noninteracting electrons $(U=0), Z=1 /[1+(4 / \pi) \lambda]$, or $(1 / Z-1) / \lambda=4 / \pi$ for small $\lambda$ and $\omega_{0} \ll D$, as shown by the arrow in Fig. 2. The natural generalization of this expression for $U>0$ is $\left(Z_{0} / Z-1\right) / \lambda$, shown in Fig. 2, since $Z_{0}=1$ for $U=0$. This quantity measures how efficiently the EPI reduces the quasiparticle weight $Z$ with respect to $Z_{0}$ obtained in the absence of EPI. In a $P$-DMFT calculation it was found that the EPI very quickly becomes inefficient when $U$ is increased [10]. In contrast, allowing for $\mathrm{AF}$, we find that the EPI remains much stronger as $U$ is increased, although it is still reduced compared to the noninteracting case. These results show

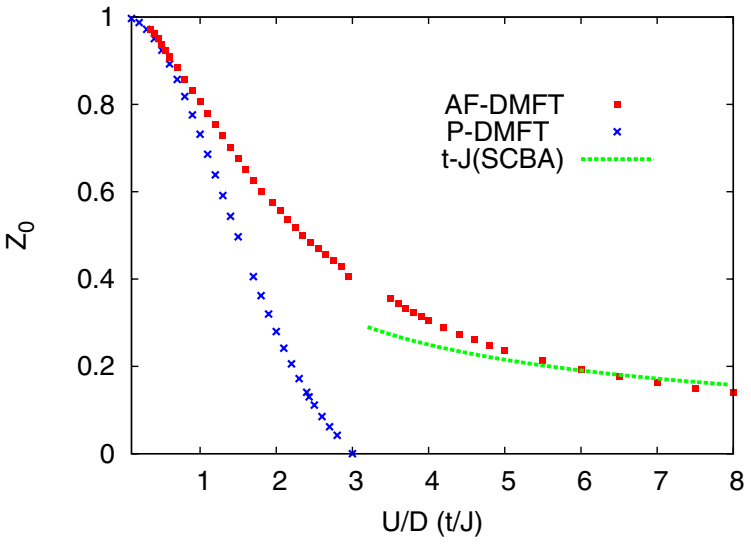

FIG. 1 (color online). Quasiparticle weight $Z_{0}$ for $\lambda=0$ for the Hubbard model (as a function of $U / D$ ) according to $P$-DMFT and AF-DMFT and for the $2 \mathrm{D} t-J$ model (as a function of $J / t)$ according to the SCBA. The figure illustrates that the AFDMFT, but not $P$-DMFT, gives reasonable values for $Z_{0}$. that AF is crucial for the EPI of the half-filled system. For $U / D$ values where the Hubbard and $t-J$ models can be compared, the AF-DMFT and the SCBA agree well for $\omega_{0}=0.025 D$. For larger phonon frequencies, however, we find that the EPI is appreciably more efficient in the SCBA than in the AF-DMFT.

We next focus on strong EPI. Figure 3 shows $Z$ as a function of $\lambda$ for different $U$. The result for $U=3.5 D$ $(J / t=0.29)$ can be compared with a calculation for the 2D $t$ - $J$ model [12] $\left(J / t=0.3\right.$ and the same $\left.\omega_{0}\right)$. As $\lambda$ is increased, $Z$ is strongly reduced, signaling polaron formation. This happens at a somewhat larger critical value $\lambda_{c}$ than was found for the $t-J$ model [12], indicated by an arrow. The deviation from Ref. [12] is probably mainly due to our use of the AF-DMFT and the neglect of "crossing" diagrams in Ref. [12]. Good agreement is also found with results for the infinite dimensional $t-J$ model [22]. These comparisons suggest that the AF-DMFT is rather accurate for the half-filled Holstein-Hubbard model.

For $U=0, Z$ drops very quickly as a function of $\lambda$ and (bi-)polarons are formed at $\lambda_{c} \approx 0.33$. As $U$ is increased the drop is slightly less rapid and polaron formation happens at somewhat larger $\lambda_{c}$. The Coulomb interaction therefore moderately suppresses polaron formation.

In $P$-DMFT calculations it was found that the effective mass $m^{*}$ depends only weakly on $\omega_{0}$ [10]. In AF-DMFT we find a sizable isotope effect on $m^{*}$ and for $\lambda \sim \lambda_{c}$ the effect is comparable to the experimental value [23].

Above we have discussed the half-filled system extensively, since we can compare with other methods and test the reliability of the AF-DMFT. The doped cuprates, however, are more interesting and challenging, and we now focus on them. Figure 4 shows $Z$ as a function of $\lambda$ for

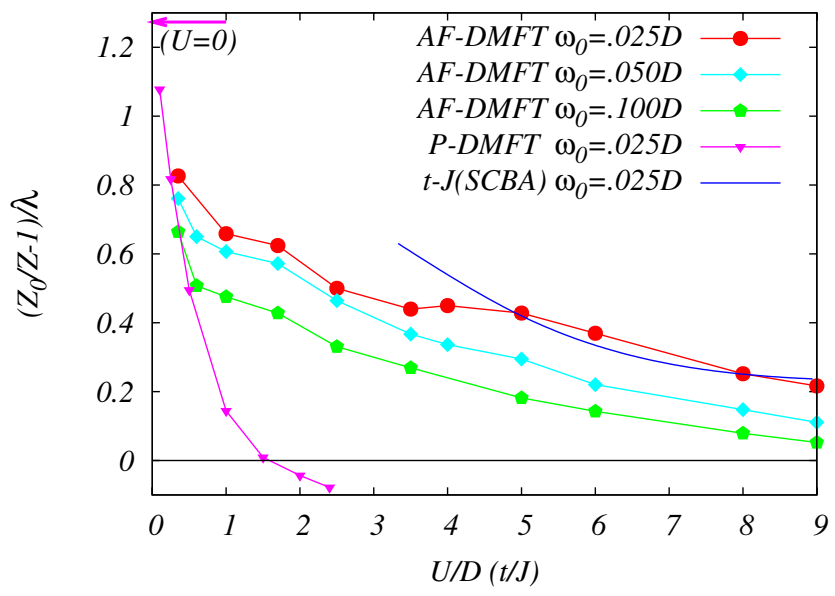

FIG. 2 (color online). $\quad\left(Z_{0} / Z-1\right) / \lambda$ in the limit $\lambda \rightarrow 0$ for the Hubbard model according to the $P$-DMFT and AF-DMFT and for the $t$ - $J$ model according to the SCBA. This quantity indicates the effectiveness of the EPI in the weak-coupling limit. The figure illustrates how the EPI is much more efficient in the AFDMFT than the $P$-DMFT, and that AF-DMFT and SCBA agree rather well. 


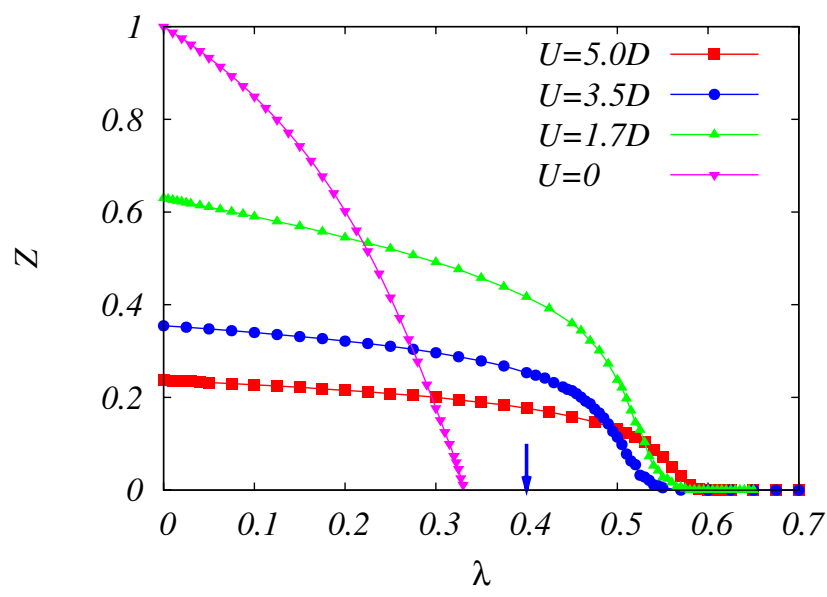

FIG. 3 (color online). $\quad Z$ as a function of $\lambda$ for different $U$ and for $\omega_{0}=0.025 D$. The arrow shows $\lambda_{c}$ of the $t-J$ model for $J / t=0.3(U / D=3.3)$ [12]. The figure shows how the Coulomb interaction moderately suppresses polaron formation $(Z \rightarrow 0)$.

$U=3.5 D$ and for different dopings. As the filling is reduced (hole doping increased) the staggered magnetization $m$ is reduced. The figure shows how this leads to an increase in $\lambda_{c}$. In a $P$-DMFT calculation [10], on the other hand, a reduction of the filling leads to a reduction of $\lambda_{c}$. The increase of $\lambda_{c}$ in the AF-DMFT with increased doping is therefore indeed due to the reduction of $m$, since at constant $m=0, \lambda_{c}$ decreases with doping.

We find the AF- $P$ transition for $U=3.5 D$ at $n=0.84$, corresponding to a much larger doping (0.16) than found experimentally. This is only partly due to our neglect of second-nearest-neighbor hopping which would introduce magnetic frustration in the system. The main reason is that in the $P$ state there are also AF correlations which lower the energy, but which are neglected in a DMFT calculation. The AF-DMFT calculation therefore favors the AF state. To obtain a balanced treatment it is necessary to use a cluster DMFT method [24,25]. Such a calculation would introduce AF correlations also in the $P$ state, and like in the AF-DMFT calculation, these correlations would weaken as the doping is increased. This should increase $\lambda_{c}$ with doping in a qualitative similar way as in Fig. 4.

Experimentally, polaron formation is found to disappear as the system is moderately doped [3]. This may be partly due to screening of the EPI, leading to a reduction of $\lambda$. However, the suppression of polaron formation with doping for fixed $\lambda$, illustrated in Fig. 4, should also be an essential part of the explanation.

Earlier work compared the Holstein $-t-J$ model with a Holstein model with one electron at the bottom of the band $[11,12]$. This is appropriate for seeing how the carrier-spin interaction influences the EPI. To study the full effects of $U$, as done here, we compare with the half-filled Holstein model, since we can then increase $U$ keeping the number of electrons unchanged. For a half-filled 2D Holstein model this is difficult, due to Fermi surface nesting and phase

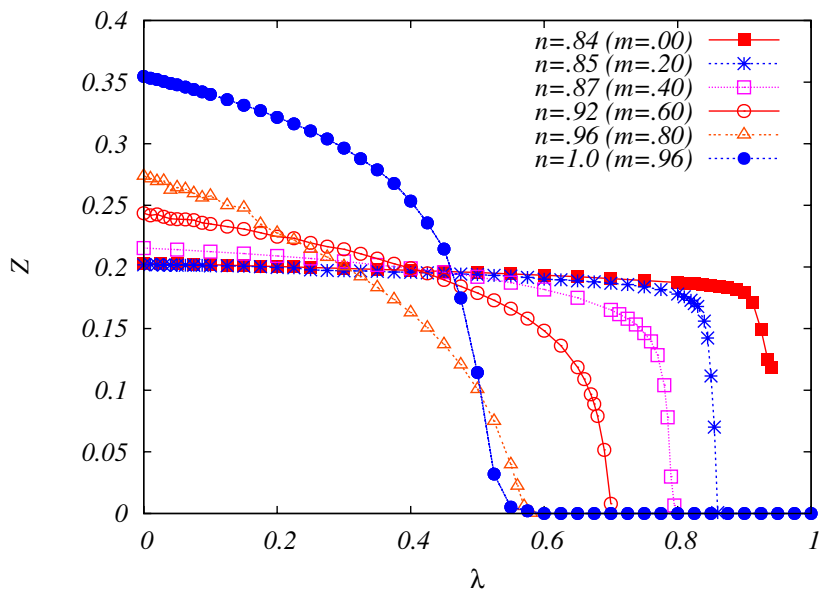

FIG. 4 (color online). $\quad Z$ as a function of $\lambda$ for different fillings $n$ and associated magnetic moments $m$ for $U=3.5 D$ and $\omega_{0}=$ $0.025 D$. The figure illustrates how the critical $\lambda_{c}$ is increased as the filling is reduced (doping is increased) due to a reduction of the antiferromagnetic correlations.

transitions. In DMFT calculations using a Bethe lattice this is not a problem.

The EPI appears much stronger for the half-filled Holstein model than for the single electron case. Comparing with the half-filled case, we find that the EPI is suppressed by the Coulomb interaction, while a comparison with the single electron case indicates an enhancement.

To discuss the difference between the two cases for a weak EPI, we calculate the electron-phonon part of the electron self-energy ( $g$ and $\omega$ small, $\omega_{0} \ll D$ )

$$
\operatorname{Re} \Sigma_{\mathrm{ep}}(\omega)-\operatorname{Re} \Sigma_{\mathrm{ep}}(0)=-\alpha \frac{g^{2}}{\pi \omega_{0} D} \omega
$$

where $\alpha=1$ for the 2D single electron case, but $\alpha=4$ for the half-filled case with a semielliptical DOS. This large difference is partly due to the DOS of the 2D Holstein model being smallest at the bottom of the band, and partly due to $\operatorname{Re} \Sigma_{\text {ep }}$ having contributions both from higher and lower states for the half-filled case, but only from higher states for the single electron case.

To understand the difference for a strong EPI, we study polaron formation in the adiabatic limit by comparing states with free electrons and perfectly localized electrons [26]. We find $E_{\text {free }}=-\beta D$ per electron, where $\beta=1$ (one electron) or $\beta=4 /(3 \pi) \approx 0.42$ (half-filled case), and $E_{\mathrm{loc}}=-g^{2} / \omega_{\mathrm{ph}}$ per electron for both cases. We assume that polarons form when $\left|E_{\text {loc }}\right|>\left|E_{\text {free }}\right|$. This leads to a large $\lambda_{c}=1$ [27] for a single electron and a much smaller $\lambda_{c}=0.42$ for the half-filled case [29].

We emphasize the remarkably small value, $\lambda_{c}=0.33$, for polaron formation in a half-filled Holstein model, meaning that Migdal's theorem breaks down for quite small $\lambda$. Using different values for $\omega_{0}$, small $\lambda_{c}$ have 

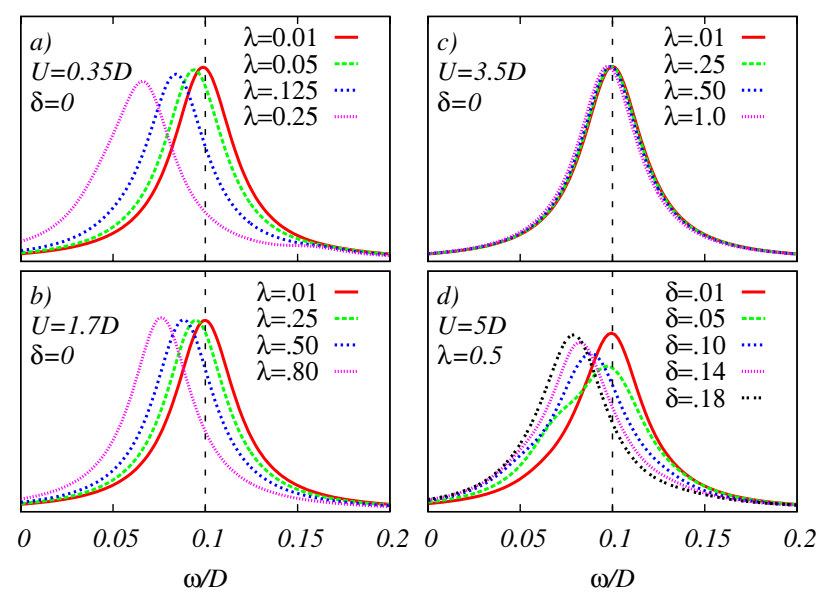

FIG. 5 (color online). Phonon spectral function for different values of $\lambda$. The bare phonon frequency is $\omega_{0}=0.1 D$ and a Lorentzian broadening with the full width at half maximum of $0.04 D$ has been introduced. (a) - (c) show how the phonon softening at half filling is dramatically suppressed by $U$ and (d) shows that the softening increases with doping $\delta$.

also been obtained earlier [30-33] using DMFT calculations.

We now consider the influence of the EPI on phonons. Figure 5(a) shows the phonon spectral function for a small $U$ at half filling (doping $\delta=0$ ) for different EPI strengths. The figure illustrates how the phonon is softened substantially as the EPI is increased. Figure 5(b) and 5(c) show that this softening is strongly reduced when $U$ is increased. The reason is that for large $U$ charge fluctuations are strongly suppressed, and the system can only respond weakly to a phonon which couples to the net charge on the atoms. Figure 5(d) shows how the softening increases as the doping is increased, due to the doped holes responding to phonons, in agreement with neutron scattering data [2]. While the EPI influences the electron self-energy strongly for large $U$ and $\delta=0$ (Fig. 2 and 3), the influence is weak for the phonon self-energy [Fig. 5(c)]. The reason is that the electron self-energy measures the response of the system to the removal or addition of a charge, which leads to a strong response even in cases where charge fluctuations are otherwise suppressed.

While paramagnetic DMFT calculations for the Holstein-Hubbard model show that effects of the EPI on electrons (quasiparticle weight) are very strongly suppressed by the Coulomb interaction, we find that this suppression is only moderate when AF correlations are included. As the doping is increased, the AF is reduced and the EPI is more suppressed. In contrast, at half filling, the Coulomb interaction strongly suppresses effects of the
EPI on phonons (phonon softening), while the suppression is reduced with doping. These trends are consistent with experiment.

We acknowledge useful discussions with S. Ciuchi and financial support (C.C. and M.C.) of Italian MIUR through the Cofin 2005 program. Calculations were performed on the Jülich JUMP computer under Grant No. JIFF22.

[1] A. Damascelli et al., Rev. Mod. Phys. 75, 473 (2003).

[2] L. Pintschovius, Phys. Status Solidi B 242, 30 (2005).

[3] K. M. Shen et al., Phys. Rev. Lett. 93, 267002 (2004).

[4] F. C. Zhang and T.M. Rice, Phys. Rev. B 37, R3759 (1988).

[5] O. Rösch and O. Gunnarsson, Phys. Rev. Lett. 92, 146403 (2004).

[6] W. Metzner and D. Vollhardt, Phys. Rev. Lett. 62, 324 (1989); M. Jarrell, Phys. Rev. Lett. 69, 168 (1992).

[7] A. Georges et al., Rev. Mod. Phys. 68, 13 (1996).

[8] G. Sangiovanni et al., Phys. Rev. Lett. 94, 026401 (2005).

[9] M. Capone et al., Phys. Rev. Lett. 92, 106401 (2004).

[10] G. Sangiovanni et al., Phys. Rev. B 73, 165123 (2006).

[11] A. Ramsak et al., Phys. Rev. B 46, R14 305 (1992).

[12] A. S. Mishchenko and N. Nagaosa, Phys. Rev. Lett. 93, 036402 (2004).

[13] T. A. Costi, Phys. Rev. Lett. 85, 1504 (2000).

[14] W. Hofstetter, Phys. Rev. Lett. 85, 1508 (2000).

[15] G. Sangiovanni et al., Phys. Rev. B 73, 205121 (2006).

[16] P. B. Allen, Phys. Rev. B 6, 2577 (1972).

[17] O. Rösch and O. Gunnarsson, Phys. Rev. Lett. 93, 237001 (2004).

[18] J.E. Han and O. Gunnarsson, Phys. Rev. B 61, 8628 (2000).

[19] Q. Si et al., Phys. Rev. Lett. 72, 2761 (1994).

[20] M. Caffarel and W. Krauth, Phys. Rev. Lett. 72, 1545 (1994).

[21] E. Dagotto, Rev. Mod. Phys. 66, 763 (1994).

[22] E. Cappelluti and S. Ciuchi, Phys. Rev. B 66, 165102 (2002).

[23] R. Khasanov et al., Phys. Rev. Lett. 92, 057602 (2004).

[24] M. H. Hettler et al., Phys. Rev. B 58, R7475 (1998).

[25] G. Kotliar et al., Phys. Rev. Lett. 87, 186401 (2001).

[26] M. Capone et al., Phys. Rev. B 56, 4484 (1997).

[27] This is in fairly good agreement with $\lambda_{c}=1.2$ from a diagrammatic Monte-Carlo calculation $\left(\omega_{0}=0.025 D\right)$ [12] or $\lambda_{c}=0.84$ from a DMFT calculation $\left(\omega_{0}=0\right)$ [28].

[28] S. Ciuchi et al., Phys. Rev. B 56, 4494 (1997).

[29] This is rather close to $\lambda_{c}=0.33$ for $U=0$ in Fig. 3 .

[30] D. Meyer et al., Phys. Rev. Lett. 89, 196401 (2002).

[31] J. E. Han et al., Phys. Rev. Lett. 90, 167006 (2003).

[32] M. Capone and S. Ciuchi, Phys. Rev. Lett. 91, 186405 (2003).

[33] M. Capone et al., Phys. Rev. B 74, 045106 (2006). 